\title{
Reduction of Large-Scale Dynamical Systems by Extended Balanced Singular Perturbation Approximation
}

\author{
Santosh Kumar Suman \\ Department of Electrical Engineering, \\ Madan Mohan Malaviya University of Technology, Gorakhpur, Uttar Pradesh, India. \\ Corresponding author: sksumanee@gmail.com \\ Awadhesh Kumar \\ Department of Electrical Engineering, \\ Madan Mohan Malaviya University of Technology, Gorakhpur, Uttar Pradesh, India. \\ E-mail: awadhesg26@gmail.com
}

(Received December 31, 2019; Accepted May 9, 2020)

\begin{abstract}
A simplified approach for model order reduction (MOR) idea is planned for better understanding and explanation of large- scale linear dynamical (LSLD) system. Such approaches are designed to well understand the description of the LSLD system based upon the Balanced Singular Perturbation Approximation (BSPA) approach. BSPA is tested for minimum / non-minimal and continuous/discrete-time systems valid for linear time-invariant (LTI) systems. The reduced-order model (ROM) is designed to preserved complete parameters with reasonable accuracy employing MOR. The Proposed approach is based upon retaining the dominant modes (may desirable states) of the system and eliminating comparatively the less significant eigenvalues. As the ROM has been derived from retaining the dominant modes of the large- scale linear dynamical stable system, which preserves stability. The strong aspect of the balanced truncation (BT) method is that the steady-state values of the ROM do not match with the original system (OS). The singular perturbation approximation approach (SPA) has been used to remove this drawback. The BSPA has been efficaciously applied on a large-scale system and the outcomes obtained show the efficacy of the approach. The time and frequency response of an approximated system has been also demonstrated by the proposed approach, which proves to be an excellent match as compared to the response obtained by other methods in the literature review with the original system.
\end{abstract}

Keywords- MOR, Large-scale linear dynamical system, Balanced truncation method, Steady state value, Singular perturbation approximation.

\section{Introduction}

The major issue in any aspect of a higher dimension systems dynamic behaviour is all over and occurs in different fields, including some engineering applications, e.g. Electrical Power system, control engineering, system design, smart city, transportation device and ecological systems, etc. ( Sikander and Prasad, 2015; Sambariya and Sharma, 2016; Daraghmeh and Qatanani, 2018; Suman and Kumar, 2019). The complex large-scale mathematical system must contain a comprehensive description of the original system (Daraghmeh et al., 2019). This mainly consists of many forms of differential and algebraic equations (Sikander and Prasad, 2015; Kumar et al., 2019). Conventional numerical modelling procedures cannot maintain the various queries as per necessity in applications that are, where the large system must be frequently used to find the solution. It is quite common that after several days, one solution has not yet been obtained. The reduction of the largescale LTI system using MOR has proven to be quite promising to get a stable solution much more quickly. The MOR is a key branch of complex system and control concept, that studies the characteristics of LSLD systems in the need to reduce the trouble while retaining their input-output behaviour intact (Chaturvedi, 2018). The key objective of MOR is to replicate the significant 
International Journal of Mathematical, Engineering and Management Sciences

Vol. 5, No. 5, 939-956, 2020

https://doi.org/10.33889/IJMEMS.2020.5.5.072

features of the actual system as closely as possible with the ROM comparison, indicating similar behaviours of the actual system. A ROM that precisely represents the prevailing characteristics of the structure under consideration is helpful and sometimes essential. Thus, an increasing essential exists for a systematic procedure to derive a lower order model, which may be called a ROM from the original system such that it preserves almost all essential properties of the system.

Numerous MOR approaches offered have been mostly in two ways that are in the shape of timedomain (TD) and frequency-domain (FD) (Sandberg and Rantzer, 2004). The TD is a form of state space as equation (2) and the FD method associated with transfer function matrix representation (3). They are divided into two basic groups of the system such as the single- input/ output (SISO) and multiple-input/output (MIMO) system (Narwal and Prasad, 2016). Reduction techniques that are used by the researchers have several advantages and disadvantages that are associated with them, as described in the literature search. Also, several common weaknesses along with the methods are that the ROM will become unstable even though the HOS remains stable (Gupta et al., 2019). Moreover, the steady-state matching of the OS with its lower order representation fails most of the time. They are furthermore hampered as the typical high-frequency ranges have poor precision and may have non-minimal phase characteristics (Davison, 1966; Cao et al., 2019) has introduced the first method for MOR in 1966, which re-modified by (Chidambara, 1969; Shamash, 1974) was suggested the Pade approximation method but these reduction methods used by researchers have several pros and cons linked to them. One of the key disadvantages of the methods is that even though the actual system is stable, the ROM is unstable (Hwang and Wang, 1984; Gupta et al., 2019) Also, a variety of approaches have been suggested to approximate large scale system as ROMs, such as Routh approximations (Hutton and Friedland, 1975), stability equation method (Pal, 1983), Mihiailov stability criterion (Wan, 1981), Chebyshev polynomial techniques (Bistritz and Langholz, 1979; Singh et al., 2012), Routh stability array (Pal, 1979), factor division method (Lucas, 1986; Parmar et al., 2007), Hurwitz polynomial approximation (Qin and Cheng, 2003), differentiation method (Bui-Thanh et al., 2008) and truncation method (Prasad et al.,1987) has been stated time to time for the desired response. (Shamash, 1981) have measured the outcome of ROM, with Markov parameter inclusive of the time moment matching to confirm the step response matching. Different methods are based on minimal realization (Lal et al., 1975) which have been suggested and that principle was extended by the Silvermans algorithm (Parthasarathy and Singh, 1975). Moreover, the principles of Markov parameters and time moments matching were used to get the ROMs for SISO systems and MIMO systems (Pal, 1983; Shamash, 1975). The main disadvantage of these methods, however, is the fact that a measure of approximate models produced for multi-systems can be equivalent to, or even more than, by which of the OS ( Pal and Ray, 1980).

Moreover, several researchers introduced the modified version continued fraction method is reported in (Parthasarathy and Jayasimha, 1982) Routh-Pade approximation (Singh et al., 2008) and the new combined method is introduced by the author (Prajapati and Prasad, 2019a). The BT, reduced system obtained through a balanced realized model subsequently elimination of less controllable and less observable states. It has been found that the model so obtained does not retain the steady-state and DC gain of the LSLD system. (Fernando and Nicholson, 1982a) suggested that weak sub-system elimination can be utilized to preserve the DC gain of the balanced truncation method using the singular perturbation approximation approach (Glover, 1984; Al-Saggaf and Franklin, 1988; Liu and Anderson, 1989; Benner and Schneider, 2010; Tiwari and Kaur, 2018). The BT model has defined the DC gain of a minimal system using a SPA that can be used when the system is stable, minimally, and internally balanced to be reduced. In this paper, the investigator 
International Journal of Mathematical, Engineering and Management Sciences

Vol. 5, No. 5, 939-956, 2020

https://doi.org/10.33889/IJMEMS.2020.5.5.072

has proposed a hybrid approach with BT and SPA approaches applicable to LSLD system with excellent DC gain matching. The benefit of the approach lies not only to the matching of steady state but its applicability to large-scale dynamical system as well, which has been confirmed over several test systems taken from literature.

\section{Proposed Methodology for System Reduction \\ 2.1 Problem Formulation}

Supposing an LTI SISO system having order ' $n$ ' is given as

$$
\left.\begin{array}{l}
\frac{d x(t)}{d(t)}=A_{o} x(t)+B_{o} u(t), t>0, x(0)=x_{0} \\
y(t)=C_{o} x(t)+D_{o} u(t), t \geq 0
\end{array}\right\} \Leftrightarrow \sum:=\left(\begin{array}{l:l}
A_{o} & B_{o} \\
\hdashline C_{o} & D_{o}
\end{array}\right)
$$

where, $x \in \mathbb{R}^{n}, u \in \mathbb{R}^{p}, y \in \mathbb{R}^{q}$, with $\mathrm{p}$ inputs and q outputs, correspondingly $\left(A_{o}, B_{o}, C_{o}, D_{o}\right)$ are constant matrices of proper size. the problem of statement for MOR of the higher-order system (HOS) with a comprehensive operational way has been introduced (Moore, 1981; Datta, 2004).

$$
\begin{aligned}
& G_{o}(s)=C_{o}\left[s I_{n}-A_{o}\right]^{-1} B_{o}+D_{o} \\
& G_{o}(s)=\frac{N_{o}(s)}{D_{o}(s)}=\frac{n_{m} s^{m}+n_{m-1} s^{m-1}+\cdots+n_{1} s+n_{0}}{d_{n} s^{n}+d_{n-1} s^{n-1}+\cdots+d_{1} s+d_{0}}
\end{aligned}
$$

where $m$ is less than $n$ and numerator polynomial $\left(n_{i}\right)$ are constants coefficient and denominator polynomial $\left(d_{i}\right)$ of the original system, correspondingly it is supposed that state of the system is such as all the roots lie in the left side of the s-map.

The difficulty lies in finding an equation (6), which in some way has approximated the original system and preserves essential parameters of the original system. Such a solution corresponds as closely as possible to the solution of the system for a similar form of input (Antoulas et al., 2018).

The MOR problem consists of finding an approximate system of the reduced system described via

$$
\left.\begin{array}{l}
\begin{array}{l}
\frac{d x(t)}{d(t)}=A_{r} x(t)+B_{r} u(t), t>0, x_{r}(0)=x_{0} \\
y(t)=C_{r} x(t)+D_{r} u(t), t \geq 0
\end{array}
\end{array}\right\}: \sum_{r}=\left(\begin{array}{l:l}
A_{r} & B_{r} \\
\hline C_{r} & D_{r}
\end{array}\right)
$$

where, $r \ll n, G_{r}(s)$ of the ROM in the form of a transfer function of HOS, is well-defined by

$$
\begin{aligned}
& G_{r}(s)=R_{r}(s) \\
& G_{r}(s)=\frac{\hat{N}_{r}(s)}{\hat{D}_{r}(s)}=\frac{\hat{n}_{r} s^{r}+\hat{n}_{r-1} s^{r-1}+\cdots+\hat{n}_{1} s+\hat{n}_{0}}{\hat{d}_{r} s^{r}+\hat{d}_{r-1} s^{r-1}+\cdots+\hat{d}_{1} s+\hat{d}_{0}}
\end{aligned}
$$


of order $\mathrm{r}, \mathrm{r}$ is very less than $\mathrm{n}$, which is approximates OS and $\hat{n}_{i}, \hat{d}_{i}$ are constants coefficient of numerator polynomial and denominator polynomial of the ROM. These parameters are to be obtained while MOR of an OS to ROM, such that the important properties of OS to preserved with less amount of error (Prajapati and Prasad, 2019b).

\subsection{Proposed Hybrid Technique for Approximation}

In this section, we address the order reducing procedure for higher-dimensional systems resulting in a hybrid approach using BT and balanced SPA. In the BT method, all balanced systems are separated into two parts as a slow and fast mode by defining the lower Hankel singular values (HSV) as fast mode, with the others defined as a slow mode. First, the derivative of all states equal to zero in fast mode can be obtained by defining a reduced system. The main aim of structure preservation in the ROM is to preserve dominant frequencies of the original system. Hence, to preserve dominant dynamic modes in the reduced system.

\subsection{The Steps of Order Reduction Algorithm using the Balanced Truncation Method to be Followed.}

Steps: In this step, both Grammians such as controllability and observability of the OS are determined as follows:

Instead of calculating the two Grammian via integral equations, solve the Lyapunov equations (9) and (10) to obtain the controllability Grammian $\left(P_{c}\right)$ and observability Grammian $\left(P_{o}\right)$.

If the eigenvalues of system matrix ' $A_{o}$ ' are implicit to strictly in left half-map then $\left(P_{c}\right)$ and $\left(P_{o}\right)$ are defined as

$$
\begin{aligned}
& P_{c}=\int_{0}^{\infty} e^{A_{o} \tau} B_{o} B_{o}{ }^{T} e^{A_{o}^{T} \tau} d \tau \\
& P_{o}=\int_{0}^{\infty} e^{A_{o}^{T} \tau C_{o}{ }^{T} C_{o} e^{A \tau} d \tau}
\end{aligned}
$$

Assumption. The $\mathrm{n}^{\text {th }}$-order dimensional system is an asymptotically stable system and also minimal. Moreover, the state-space system equation (1) or the pair $\left(A_{o}, B_{o}\right)$ states controllable if and only if the $\mathrm{n} \times \mathrm{nm}$ state controllability matrix and pair $\left(A_{o}, C_{o}\right)$ are observable if the $n p \times n$ observability matrix (Moore, 1981).

By assumption, both Grammians $P_{c}$ and $P_{O}$ are a positive definite and unique symmetric matrix explanation to the couple of Grammians. Since their implementation is minimal.

- These both Grammians satisfy the following linear Lyapunov equations (Gugercin and Antoulas, 2004; Imran et al., 2014).

$$
\begin{aligned}
& A_{o} P_{c}+P_{c} A_{o}^{T}+B_{o} B_{o}^{T}=0 \\
& A_{o}^{T} P_{o}+P_{o} A_{o}+C_{o} C_{o}^{T}=0
\end{aligned}
$$


- In control philosophy, eigenvalues express system stability, although HSV describes the "energy" of each state in the system.

- Numerically we express as a stable state-space system equation (1), its HSV is well-defined as $\sigma_{H S V}=\sqrt{\lambda_{i}\left(P_{c} P\right)}$ respectively. For simplicity, such singular values (SV) are generally ordered downward to truncate states that match smaller Hankel singular values as follows $\sigma_{1} \geq \sigma_{2} \geq \sigma_{3} \geq \sigma_{4} \geq \sigma_{r} \geq \sigma_{r+1} \geq \cdots \sigma_{n}>0$ (Sikander and Prasad, 2017).

- This is also a significant action of the minimality of realization of the original system is the diminishing positive number such that

$$
P_{c}=P_{o}=\sum=\text { Diagonal }\left\{\sigma_{1}, \sigma_{2}, \sigma_{3}, \sigma_{4} \cdots \sigma_{n}\right\}
$$

- The diagonal matrix $\left(\sum\right.$ ) if such a matrix realization exists (Lall et al., 2002; Gugercin and Antoulas, 2004; Segalman, 2007).

$$
\sigma(\omega)=\left\|G_{o}(j \omega)\right\| 2
$$

- Compute (Cholesky) factors (CF) of the Grammians are often obtained by this factorization according to (Pernebo and Silverman, 1982; Datta, 2004). The lower triangular matrix (CF) $Q_{c}$ and $Q_{o}$ of both Grammians $P_{c}$ and $P_{o}$ is obtained as (Al-Saggaf and Franklin, 1988; Datta, 2004).

$$
P_{c}=Q_{c} Q_{c}^{T}
$$

$$
P_{o}=Q_{o} Q_{o}^{T}
$$

- Compute SVD, the $Q_{o}^{T} Q_{c}$ is singular value decomposition of Grammians, also known as SVD of the system, found as follows (Datta, 2004).

$$
\operatorname{SVD}\left(Q_{o}^{T} Q_{c}\right)=U \sum V^{*}
$$

where, $U$ and $V$ are a vector, define as left and right singular. Also, unitary matrices (orthogonal). This system matric can be transformed into the balanced model by a similarity transformation matrices W, which can be achieved as follows (Datta, 2004; Gugercin, 2008; Imran et al., 2014). $\mathrm{ROM}$ is $\left(W A W^{-1}, W B, C W^{-1}\right)$, where $\mathrm{W}$ is a transformation matrix

$$
W=Q_{c} V \sum^{-\frac{1}{2}}
$$

The original system has been completely balanced, which is partitioned as: 
International Journal of Mathematical, Engineering and Management Sciences

Vol. 5, No. 5, 939-956, 2020

https://doi.org/10.33889/IJMEMS.2020.5.5.072

$G_{B a l}(s)=\left[\begin{array}{c:c}W A W^{-1} & W B \\ \hline C W^{-1} & D\end{array}\right]:$ Balanced System

$=\underbrace{\left[\begin{array}{c:c}A_{B} & B_{B} \\ \hline C_{B} & D_{B}\end{array}\right]}_{\text {Balanced System }} \Leftrightarrow \sum:\left[\begin{array}{cc}\Sigma_{1} & 0 \\ 0 & \Sigma_{2}\end{array}\right]$

Since partition, the balanced system $\left(A_{B}, B_{B}, C_{B}, D_{B}\right)$, and the Grammian $\sum$ conformally given as

$$
\left.\begin{array}{rl}
A_{B} & \left.=\left[\begin{array}{ll}
A_{11} & A_{12} \\
A_{21} & A_{22}
\end{array}\right], B_{B}=\left[\begin{array}{l}
B_{1} \\
B_{2}
\end{array}\right],\right\}: \underbrace{\text { Strong Subsyustem }}_{\text {(to be retained) }}+\underbrace{\text { Weak Subsystem }}_{\text {(to be retained) }} \Leftrightarrow \sum \\
C_{B} & =\left[\begin{array}{ll}
C_{1} & C_{2}
\end{array}\right], D_{B}=D
\end{array}\right] \sum
$$

where, $A_{11}$ is part of a strong subsystem and $\sum_{1}$ are $r \times r(r<n)$ matrixes.

We call this reduced order model a balanced system approximation of direct-truncation (DT). There are some well-known results on approximation ( Liu and Anderson, 1989).

Lemma A. (Pernebo and Silverman, 1982)

The subsystem matrix $\left(A_{i i}, B_{i}, C_{i}\right)$ is the minimal and internally balanced realization through Grammian $\sum_{i}(i=1,2)(\mathrm{i}=1,2)$.

Lemma B. (Pernebo and Silverman, 1982)

the subsystem matrix $A_{i i}(i=1,2)$ is asymptotically stable if $\sum_{1}$ and $\sum_{2}$ has no common diagonal component. Furthermore, the subsystem $\left(A_{11}, B_{1}, C_{1}\right)$ for $(i=1,2)$ is both completely controllable and observable (Fernando and Nicholson,1982a).

Now, let us focus on applying of the SPA to the reduced model reduction of an LTI system (Fernando and Nicholson, 1982b).

Equation (19) has been attained as a minimal realised model containing strong and weakly subsystems. Thus, the SPA may be effortlessly applied to subsystems of Equation (19). In the BT model, reduced ( $r$ ) balanced states are retained which are completely controllable and observable so balanced states are preserved, and remaining weakly controllable and/or observable states are truncated. SPA is used to maintain the DC gain of the original system in the model (Safonov and Chiang, 1989; Kumar et al., 2012). The concerned researcher may referee to (Kokotovic et al., 1976) for more indications of the method.

As the balanced realised system determine, can be re-write in the form of given as 


$$
\begin{aligned}
& {\left[\begin{array}{l}
\frac{d x_{1}}{d t} \\
\frac{d x_{2}}{d t}
\end{array}\right]=\left[\begin{array}{ll}
A_{11} & A_{12} \\
A_{21} & A_{22}
\end{array}\right]\left[\begin{array}{l}
x_{1} \\
x_{2}
\end{array}\right]+\left[\begin{array}{l}
B_{1} \\
B_{2}
\end{array}\right] u(t)} \\
& \left.\begin{array}{l}
y=\left[\begin{array}{ll}
C_{1} & C_{2}
\end{array}\right]\left[\begin{array}{l}
x_{1} \\
x_{2}
\end{array}\right]+D u(t)
\end{array}\right\}: \text { Balanced Model }
\end{aligned}
$$

Again, re-write is equation form

$$
\frac{d \dot{x}_{1}}{d t}=A_{11} x_{1}+A_{12} x_{2}+B_{1} u(\text { Slower })
$$

$\mu \frac{d \dot{x}_{2}}{d t}=A_{21} x_{1}+A_{22} x_{2}+B_{2} u($ Faster $)$

where, $\mu$ is a positive small perturbational parameter of singular perturbation approximation approach (Fernando and Nicholson, 1983; Gajic and Lelic, 2001).

By comparing the derivative of the weakly subsystem to zero below, the BSPA model can be achieved (Kumar et al., 2012; Guiver, 2019).

Now the final system $\left(\hat{A}_{B S P A}, \hat{B}_{B S P A}, \hat{C}_{B S P A}, \hat{D}_{B S P A}\right)$ conformally as in (23).

$$
G_{B S P A}=\left[\begin{array}{c:c}
\hat{A}_{B S P A} & \hat{B}_{B S P A} \\
\hdashline \hat{C}_{B S P A} & \hat{D}_{B S P A}
\end{array}\right]: \underbrace{\left[\begin{array}{c:c}
A_{11}-A_{12} \otimes A_{22}^{-1} \otimes A_{21} & B_{1}-A_{12} \otimes A_{22}^{-1} \otimes B_{2} \\
\hdashline C_{1}-C_{2} \otimes A_{22}^{-1} \otimes A_{21} & D-C_{2} \otimes A_{22}^{-1} \otimes B_{2}
\end{array}\right]}_{\text {Balanced SPA }}
$$

The various numerical tests to demonstrate the technique will be discussed in the preceding section.

\subsection{Error Investigation of Reduced-Order Models}

If the reduced system has been obtained from the original system, then the modeling error transfer function is well-defined via $E_{r}(s)=\left[G_{o}(s)-G_{r}(s)\right]$.

The actual infinity norm ( $\boldsymbol{H}_{\infty}$ ) error bound in $\boldsymbol{r}^{\text {th }}$ a ROM may be calculated by taking $\boldsymbol{H}_{\infty}$ of $E_{r}(s)$ . The actual and theoretical infinity error bounds are given by $\left\|E_{r}(s)\right\|_{\infty}$ and $\leq 2 \sum_{i=r+1}^{n=\text { order of system }} \sigma_{i}$ respectively, Furthermore, the actual amount of error bound is continuously less than or equal to the theoretical amount of error bound. Bound of the system. (Datta, 2004; Antoulas, 2005). Thus, $\left\|E_{r}(s)\right\|_{\infty}=\left\|\left[G_{o}(s)-G_{r}(s)\right]\right\|_{\infty} \leq 2 \sum_{i=r+1}^{n} \sigma_{i}$ it is also known as a Priori Error bound. 
Also, the comparison of the response has been carried out on the basis of the unit step response. The performance of ROM obtained is also compared on the basis of measured by calculating the performance indices, the accurateness of the proposed approach is known as the integral square error (ISE) between the transient sections of the actual system and the reduced-order model. The proposed scheme is used to achieve the best values of the coefficients in Equation 6., for reduce model, by diminishing the performance indices ISE, also, to evaluate the performance of the reduced model in comparison with the original system and the reduced systems in terms of integral absolute error (IAE) (Singh et al., 2004; Sikander and Prasad, 2015; Tiwari and Kaur, 2018) described by the following equation

$$
I S E=\int_{0}^{\infty}\left[y_{1}(t)-y_{2}(t)\right]^{2} d t \text { and } I A E=\int_{0}^{\infty}\left|y_{1}(t)-y_{2}(t)\right| d t
$$

\section{Numerical Experiments}

Test System 1: Consider a FOM model for successfully applied the proposed reduction method. It is a large-scale dynamical system of a benchmarks problem of order 1006, This test illustration is taken from (Chahlaoui and Van Dooren, 2002; 2005).

The state-space matrices form is given by where, $e_{i} \in \mathbb{R}^{i, 1}$ is the vector with each entry equal to 1 :

$$
\left.\begin{array}{c}
A_{o}=\left[\begin{array}{llll}
A_{1} & & & \\
& A_{2} & & \\
& & A_{3} & \\
& & & A_{4}
\end{array}\right], A_{1}=\left[\begin{array}{cc}
-1 & 100 \\
-100 & -1
\end{array}\right], A_{2}=\left[\begin{array}{cc}
-1 & 200 \\
-200 & -1
\end{array}\right], \\
A_{3}=\left[\begin{array}{ccc}
-1 & 400 \\
-400 & -1
\end{array}\right], A_{4}=-\operatorname{diag}(1,2, \cdots, 1000), B_{o}=\left[\begin{array}{c}
10 e 6 \\
e 1000
\end{array}\right], C_{o}=B_{o} T
\end{array}\right\}
$$

Figure 1 and Figure 3 have been shown, both responses, time, and frequency of the OS. The bar graph of HSV have been calculated and also plotted in Figure 2. This bar graph displays the optimum reduction order. The number of singular values that dominate non-zero is assumed to be that as an order of the original system. The first- eight singular values here are important, and then the ninth singular values quickly decay. Consequently, the order of reduction has been preferred as an eighth order. 
International Journal of Mathematical, Engineering and Management Sciences

Vol. 5, No. 5, 939-956, 2020

https://doi.org/10.33889/IJMEMS.2020.5.5.072

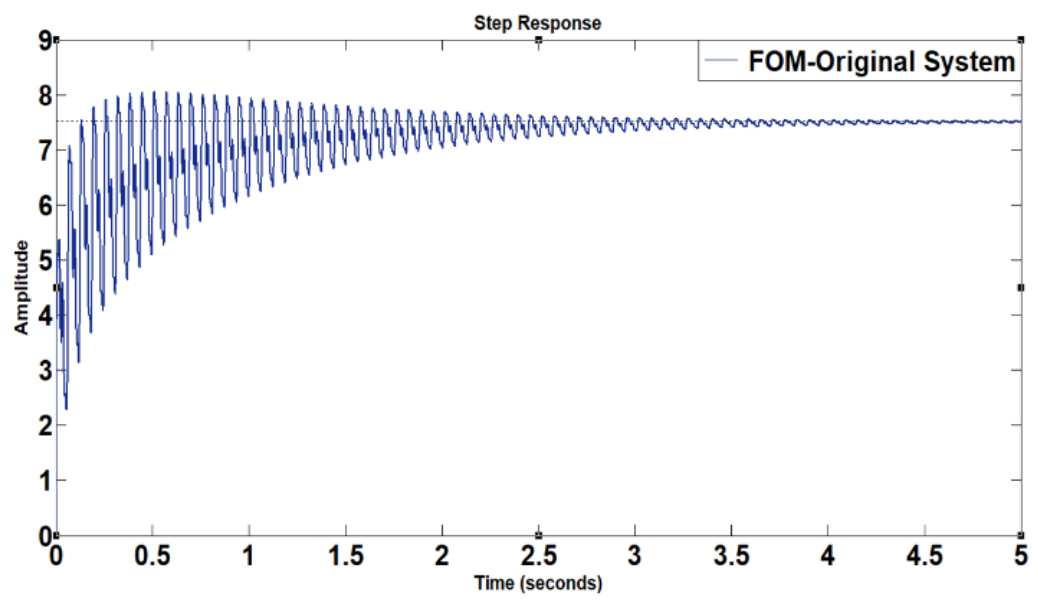

Figure 1. Time response of the OS

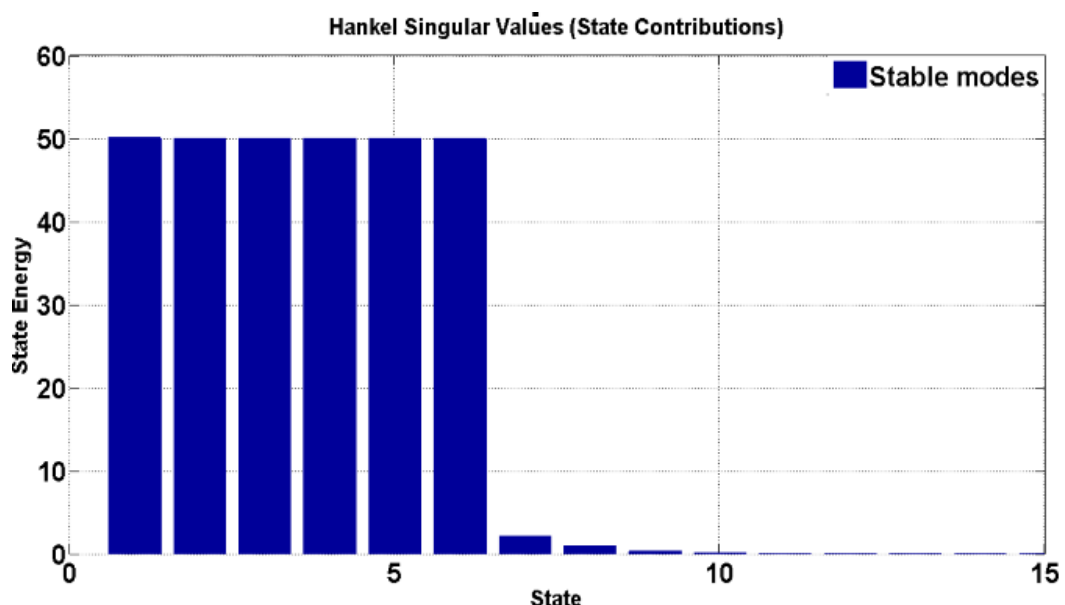

Figure 2. Bar chart Hankel singular value plot of the OS

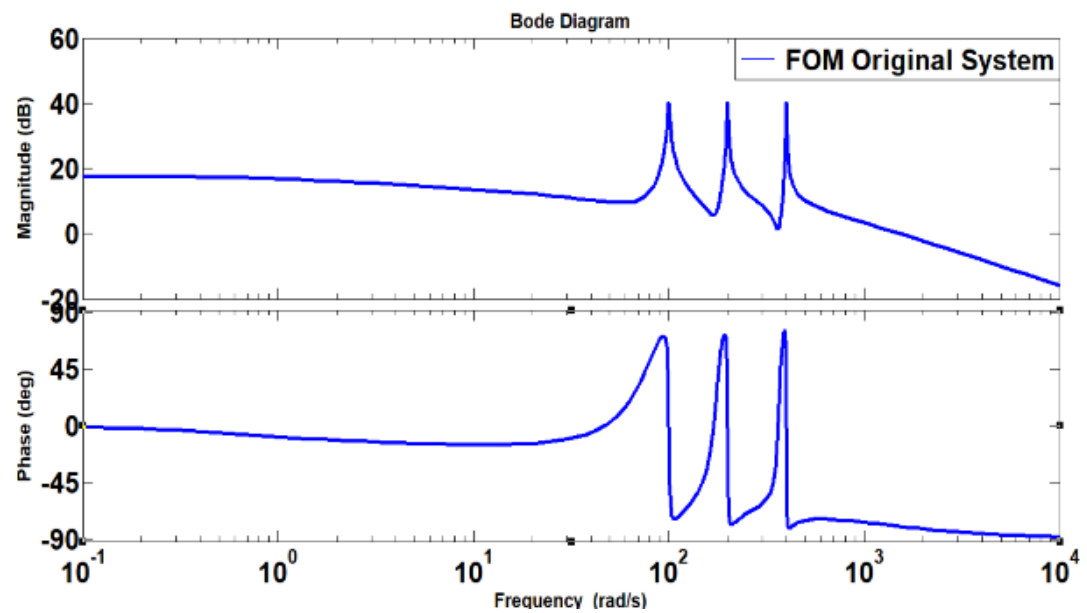

Figure 3. Frequency response of the OS 
International Journal of Mathematical, Engineering and Management Sciences

Vol. 5, No. 5, 939-956, 2020

https://doi.org/10.33889/IJMEMS.2020.5.5.072

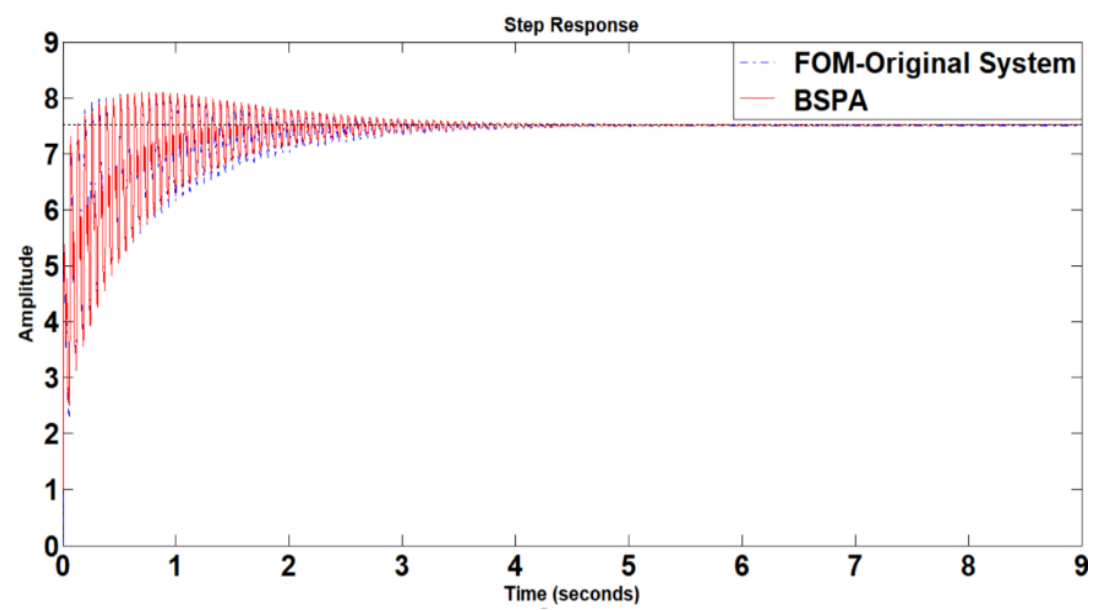

Figure 4. Time response of ROM with OS

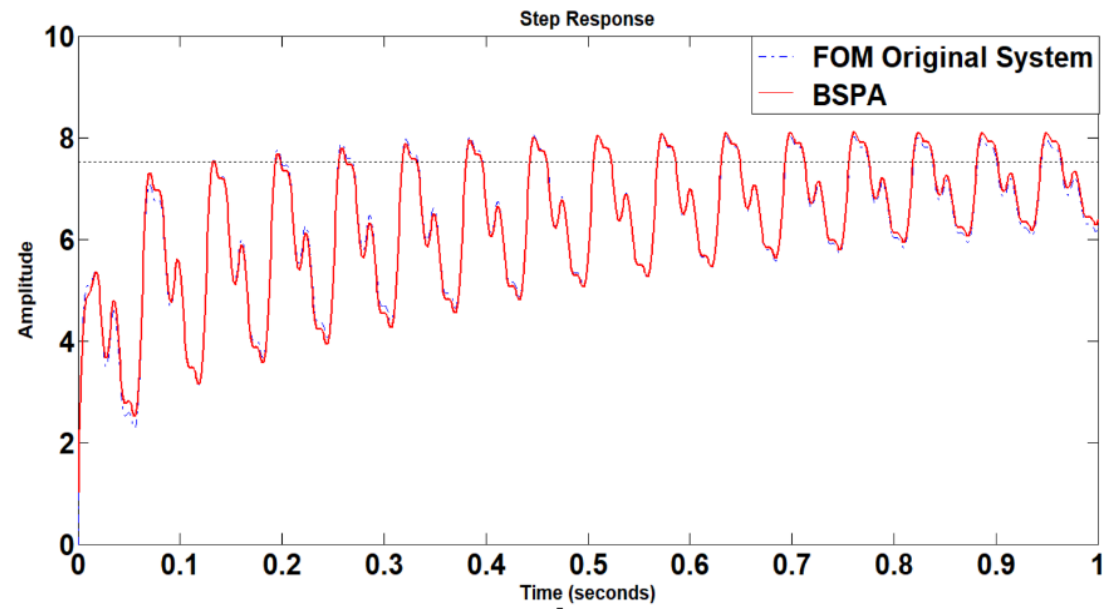

Figure 5. Time response of ROM with OS for one second

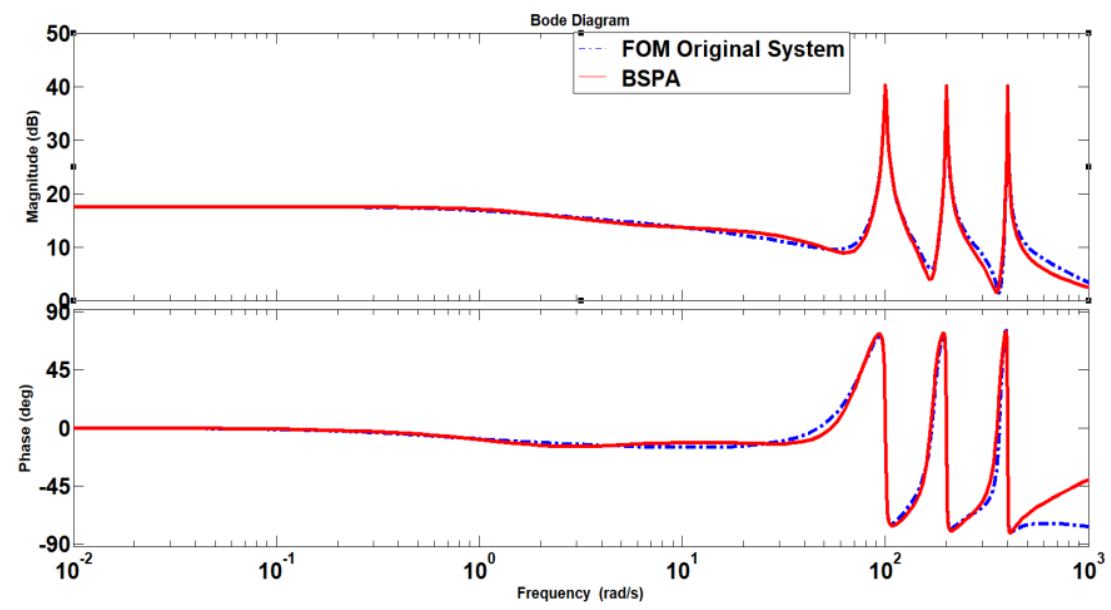

Figure 6. Frequency response of ROM with OS 
International Journal of Mathematical, Engineering and Management Sciences

Vol. 5, No. 5, 939-956, 2020

https://doi.org/10.33889/IJMEMS.2020.5.5.072

The ROM matrices obtained are given as

$\hat{A}_{B S P A}=\left[\begin{array}{cccccccc}-5.87-1.03 \mathrm{e}-25 \mathrm{i} & -1.23+1.31 \mathrm{e}-25 \mathrm{i} & -80.3-2.24 \mathrm{e}-26 \mathrm{i} & -0.359+7.43 \mathrm{e}-26 \mathrm{i} & 69.9-1.92 \mathrm{e}-27 \mathrm{i} & 180+3.28 \mathrm{e}-26 \mathrm{i} & -5.83+7.32 \mathrm{e}-28 \mathrm{i} & 3.06-1.87 \mathrm{e}-27 \mathrm{i} \\ -1.23+8.4 \mathrm{e}-26 \mathrm{i} & -0.256-2.11 \mathrm{e}-25 \mathrm{i} & -362-4.84 \mathrm{e}-26 \mathrm{i} & -0.0749-6.28 \mathrm{e}-26 \mathrm{i} & 44.5-4.59 \mathrm{e}-26 \mathrm{i} & 81.9-3.47 \mathrm{e}-26 \mathrm{i} & -1.22+4.13 \mathrm{e}-27 \mathrm{i} & 0.64-2.81 \mathrm{e}-27 \mathrm{i} \\ 80.3-1.5 \mathrm{e}-26 \mathrm{i} & 362+4.44 \mathrm{e}-26 \mathrm{i} & -0.000375-2.85 \mathrm{e}-25 \mathrm{i} & -12.9+1.17 \mathrm{e}-25 \mathrm{i} & 0.000464+1.29 \mathrm{e}-25 \mathrm{i} & 0.00149+1.47 \mathrm{e}-25 \mathrm{i} & -0.0509+8.32 \mathrm{e}-28 \mathrm{i} & 0.0255-1.07 \mathrm{e}-27 \mathrm{i} \\ -0.359-2.57 \mathrm{e}-26 \mathrm{i} & -0.0749-4.99 \mathrm{e}-26 \mathrm{i} & 12.9-8.48 \mathrm{e}-26 \mathrm{i} & -0.022-4.45 \mathrm{e}-26 \mathrm{i} & 155+3.73 \mathrm{e}-27 \mathrm{i} & 50.5+1.41 \mathrm{e}-26 \mathrm{i} & -0.356+2.04 \mathrm{e}-28 \mathrm{i} & 0.187-2.83 \mathrm{e}-28 \mathrm{i} \\ -69.9-5.52 \mathrm{e}-27 \mathrm{i} & -44.5-1.17 \mathrm{e}-28 \mathrm{i} & 0.000464-1.58 \mathrm{e}-25 \mathrm{i} & -155-1.01 \mathrm{e}-26 \mathrm{i} & -0.000573+1.92 \mathrm{e}-26 \mathrm{i} & -0.00184+3.97 \mathrm{e}-26 \mathrm{i} & 0.0628-8.1 \mathrm{e}-29 \mathrm{i} & -0.0314-9.26 \mathrm{e}-29 \mathrm{i} \\ -180+6.62 \mathrm{e}-27 \mathrm{i} & -81.9-4.72 \mathrm{e}-27 \mathrm{i} & 0.00149-5.16 \mathrm{e}-26 \mathrm{i} & -50.5-2.58 \mathrm{e}-26 \mathrm{i} & -0.00184+1.25 \mathrm{e}-26 \mathrm{i} & -0.0059+6.91 \mathrm{e}-27 \mathrm{i} & 0.202-1.4 \mathrm{e}-27 \mathrm{i} & -0.101+1.16 \mathrm{e}-27 \mathrm{i} \\ -5.83-3.5 \mathrm{e}-28 \mathrm{i} & -1.22+2.09 \mathrm{e}-27 \mathrm{i} & 0.0509+7.41 \mathrm{e}-27 \mathrm{i} & -0.356-1.36 \mathrm{e}-27 \mathrm{i} & -0.0628-2.33 \mathrm{e}-28 \mathrm{i} & -0.202-1.12 \mathrm{e}-27 \mathrm{i} & -36-1.85 \mathrm{e}-28 \mathrm{i} & 25.7+1.57 \mathrm{e}-28 \mathrm{i} \\ 3.06+7.83 \mathrm{e}-28 \mathrm{i} & 0.64-1.6 \mathrm{e}-27 \mathrm{i} & -0.0255-6.29 \mathrm{e}-27 \mathrm{i} & 0.187+1.22 \mathrm{e}-27 \mathrm{i} & 0.0314+6.87 \mathrm{e}-28 \mathrm{i} & 0.101+1.01 \mathrm{e}-27 \mathrm{i} & 25.7+1.45 \mathrm{e}-28 \mathrm{i} & -21.7-1.19 \mathrm{e}-28 \mathrm{i}\end{array}\right]$

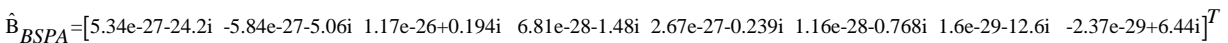



$\hat{D}_{B S P A}=[1-1.12 \mathrm{e}-28 \mathrm{i}]$

The eigenvalues of the original system are given as $\sigma\left(A_{o}\right)$ is given by

$$
\sigma\left(A_{O}\right)=[-1,-2, \ldots,-1000,-1 \pm 100 j,-1 \pm 200 j,-1 \pm 400 j]
$$

Figure 4 and Figure 6 is shown both responses, time and frequency of the original system with a ROM and also compared. It is seen that the ROM obtained by the BSPA approach, offers close approximation to the system under consideration. Furthermore, another time response of the OS and ROM for one second has been also shown in Figure 5. It is the magnification (zoom) of both systems response to views the transient behaviour comparison. Further another frequency response of the ROM with the OS is also depicted in Figure 6. It is again evidently demonstrating that the $\mathrm{ROM}$ to be a close representation of the OS.

Also, the infinity norm of the modelling error has been computed to show the effectiveness of the method. It is depicted in Table 1. It is understood that the actual error values are lower than the theoretical error values which may due to the underdamped nature of the system or due to poor transient response in an attempt to remove the steady-state error. Also, to achieve exact approximation and confirms the efficacy of the method. In addition, the response is also compared on the basis of performance indices as shown in Table 2 using the proposed approach, the ISE and IAE value is found the lowest value than another method. performance indices have been calculated to show the efficacy of the BSPA approach.

Test System 2: We now consider a Continuous-LTI and stable system of a simple mechanical system of $6^{\text {th }}$ order .for comparison purposes, this test system is taken from (Gawronski and Juang, 1990).

$$
\begin{aligned}
A_{O} & =\left[\begin{array}{lllllll}
0 & 0 & 0 & 1 & 0 & 0 & \\
0 & 0 & 0 & 0 & 1 & 0 & \\
0 & 0 & 0 & 0 & 0 & 1 & \\
-5.4545 & 4.5455 & 0 & -0.0545 & 0.0455 & 0 & \\
10 & -21 & 11 & 0.1000 & -0.2100 & 0.1100 \\
0 & 5.5000 & -6.5000 & 0 & 0.0550 & -0.0650
\end{array}\right] \\
B_{O} & =\left[\begin{array}{llllllll}
0 & 0 & 0 & 0.0909 & 0.4 & -0.5
\end{array}\right]^{T}, C_{O}=\left[\begin{array}{llllll}
2 & -2 & 3 & 0 & 0 & 0
\end{array}\right]
\end{aligned}
$$


International Journal of Mathematical, Engineering and Management Sciences

Vol. 5, No. 5, 939-956, 2020

https://doi.org/10.33889/IJMEMS.2020.5.5.072

Hankel singular values of the OS are

$$
\sigma\left(A_{O}\right)=\left[\begin{array}{llllll}
15.8456 & 15.7084 & 0.9097 & 0.8874 & 0.6271 & 0.5961
\end{array}\right] .
$$

The ROM matrices found are given as

$$
\begin{aligned}
& \hat{A}_{B S P A}=\left[\begin{array}{cc}
-0.003786 & 0.8738 \\
-0.8738 & -0.003812
\end{array}\right] \\
& \hat{B}_{B S P A}=\left[\begin{array}{ll}
-0.3464 & -0.3461
\end{array}\right]^{T}, \\
& \hat{\mathrm{C}}_{B S P A}=\left[\begin{array}{ll}
0.3464 & -0.3461
\end{array}\right], \hat{D}_{B S P A}=[-0.1066]
\end{aligned}
$$

Bar chart HSV for the original system has been calculated and depicted in Figure 8. Which is show the optimal order reduction. Relatively, the number of non-zero dominant singular values non-zero dominant is regarded as the model of the reduction scheme. The first-second singular values are most significant here and afterward, the third singular values have decayed suddenly, becoming insignificant. So, the order of reduction is obvious as the second order.

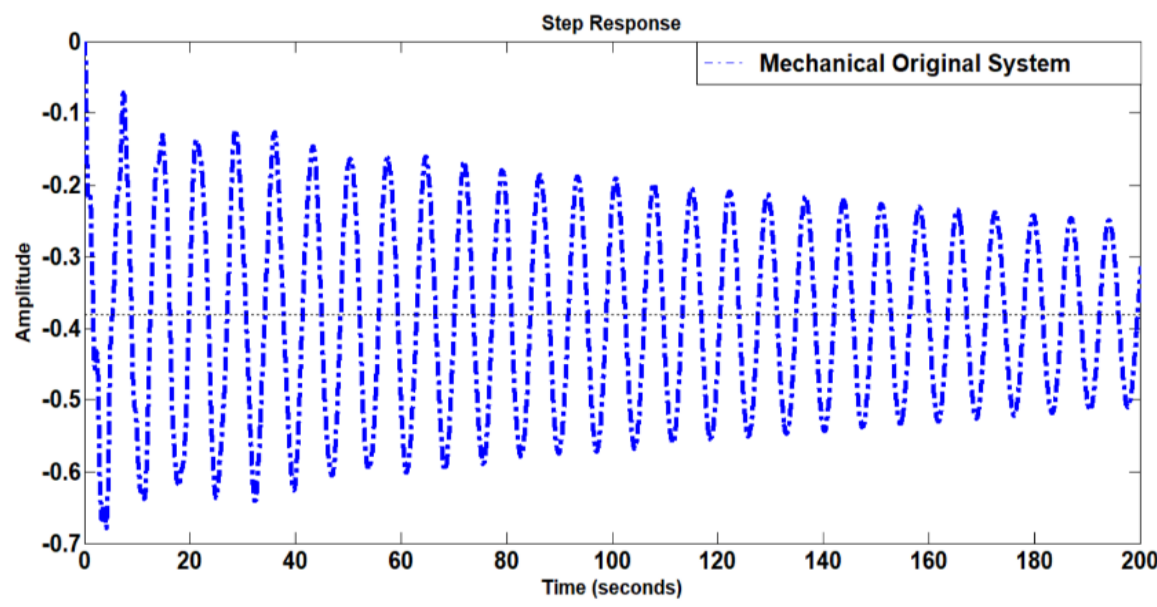

Figure 7. Time response of mechanical OS 
International Journal of Mathematical, Engineering and Management Sciences

Vol. 5, No. 5, 939-956, 2020

https://doi.org/10.33889/IJMEMS.2020.5.5.072

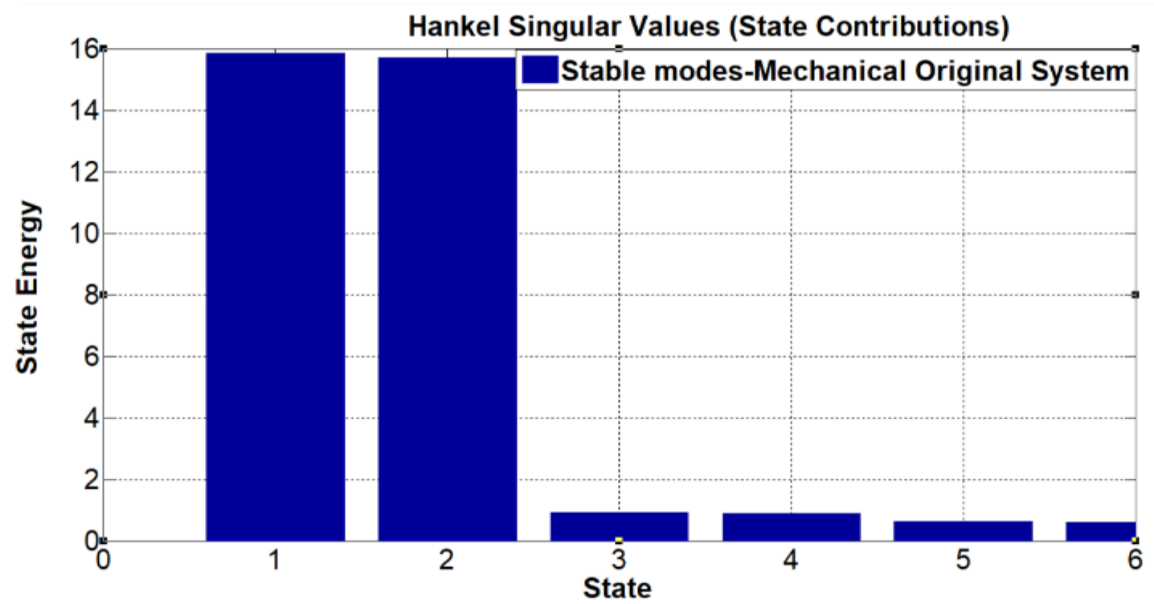

Figure 8. The bar chart in Hankel singular value plot of mechanical OS

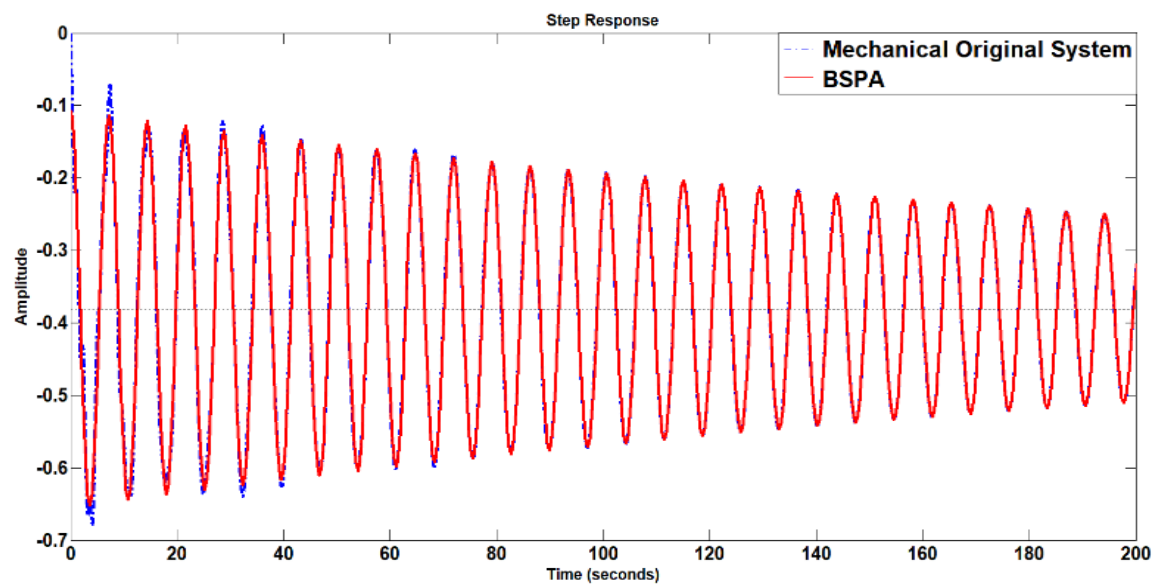

Figure 9. Time response of ROM with OS for 200s (time)



Figure 10. Frequency response of the ROM with mechanical OS 
Figure 7 and Figure 10 have shown both responses, time, and frequency of the reduced-order model with the original system and also compared. Respectively and it is understood that the response of ROM approximates the results of the OS very closely with no steady-state error in time response and exactly matches in frequency response. Furthermore, another time response of the OS and ROM for 200s second has been also shown in Figure 9. It is the magnification (zoom) of both systems response to views the transient behaviour comparison. It is again evidently Demonstrating that the reduced system to be a close characteristic with all essential parameters of the original system. Also, the infinity norm $\left(H_{\infty}\right)$ of the modelling error has been computed to show the effectiveness of the method. It is tabulated in Table 1. It is observed that the actual value of error bound is lower than the theoretical value of error bound which may due to the underdamped nature of the system or due to poor transient response in an attempt to eliminate the steady-state error. Also, to achieve exact approximation and confirms the efficacy of the method. In addition, the response is also compared on the basis of performance indices as shown in Table 2 using the proposed approach, the ISE and IAE value is found the lowest value than another method. performance indices have been calculated to show the efficacy and accuracy of the proposed approach.

\section{Result and Discussion}

In this discussion, the time and frequency responses of the ROM with OS are shown in the above figures. All mathematical experiments have been performed using Matrix Laboratory R2019a (Academic use). The time and frequency responses of the OS and ROM in Figures of the for-Test systems. This proposed method is excellent in comparison to the use of the BT method and other methods. The infinity norm of the modelling error has been also computed and results are depicted in Table 1. it is seen that to an excellent precise approximation with a minor error of the OS with the ROM. again, it is observed that the actual value of error bound is obtained lower than the theoretical value of error bound which may due to the underdamped nature of the system or due to poor transient response in an attempt to remove the steady-state error. In addition, the closeness and accuracy of the response have been measured through Performance Indices, presented in Table 2. The ISE and IAE value by the proposed approach is much lower than those compared with BT methods. This shows the superiority of the proposed approach.

Table 1. Error bound for different reduction methods for test systems

\begin{tabular}{|c|c|c|c|c|}
\hline \multirow{3}{*}{ Computations } & \multicolumn{4}{|c|}{$H_{\infty}$ Norm Values } \\
\hline & \multicolumn{2}{|c|}{ Test system 1} & \multicolumn{2}{|c|}{ Test system 2} \\
\hline & BT & Proposed Method & BT & Proposed Method \\
\hline$\left\|G_{o}(s)\right\|_{\infty} H_{\infty}$ Norm & \multicolumn{2}{|c|}{102.3342} & \multicolumn{2}{|c|}{31.5554} \\
\hline Theoretical $H_{\infty}$ Norm: $\leq 2 \sum_{i=r+1}^{n} \sigma_{i}$ & \multicolumn{2}{|c|}{$\begin{array}{l}1.00407 \\
\text { For } n=8\end{array}$} & \multicolumn{2}{|r|}{$\begin{array}{c}6.0407 \\
\text { For } n=2\end{array}$} \\
\hline $\begin{array}{l}\text { Actual infinity norm }\left(H_{\infty}\right) \\
\left\|G_{o}(s)-G_{r}(s)\right\|_{\infty} \text { Error Bound in } \\
\text { Modeling }\end{array}$ & 1.00390 & 0.841 & 1.8007 & 0.0012 \\
\hline $\begin{array}{l}\text { Actual Relative Error Bound: } \\
\text { Actual Error Bound } \\
\left\|G_{o}(s)\right\|_{\infty}\end{array}$ & 0.00981 & 0.0082181 & 0.05706 & 0.0000380 \\
\hline
\end{tabular}


International Journal of Mathematical, Engineering and Management Sciences

Vol. 5, No. 5, 939-956, 2020

https://doi.org/10.33889/IJMEMS.2020.5.5.072

Table 2. Performance analysis of proposed with other existing reduction methods for test system

\begin{tabular}{|l|l|l|l|}
\hline Test system & Model Reduction Method & ISE & IAE \\
\hline \multirow{2}{*}{ Test system 1 } & Proposed Method & 1.2710 & 3.1610 \\
\cline { 2 - 4 } & BT & 6.6168 & 9.9530 \\
\hline \multirow{2}{*}{ Test system 2 } & Proposed Method & 0.2436 & 0.0667 \\
\cline { 2 - 4 } & BT & 0.6611 & 5.3149 \\
\hline
\end{tabular}

\section{Conclusion}

In this paper, a new proposed approach of ROM of large-scale dynamic LTI systems, based upon BSPA methodology has been presented. It is superior to all of the traditional techniques or other approaches. This way weakness has been eliminated by the SPA from BT. The proposed approaches are the result of the hybridization of BT and the SPA method. This method has been used to effectively present a few test systems of the large-scale dynamic LTI systems. Furthermore, the time-frequency response of the proposed approach based on BSPA to gives ROM more precise and exact approximation than other approaches. In additional, comparing the error standard with existing works demonstrates the precision, reliability and enhanced efficiency of the presented system. This approach is more effective when successfully applied to a large-scale system.

\section{Conflict of Interest}

The authors confirm that there is no conflict of interest to declare for this publication.

\section{Acknowledgements}

The authors wish to express their appreciation to the Journal Editors and an anonymous referee whose comments and recommendations enhanced both the superiority and the presentation of the paper.

\section{References}

Al-Saggaf, U.M., \& Franklin, G.F. (1988). Model reduction via balanced realizations: an extension and frequency weighting techniques. IEEE Transactions on Automatic Control, 33(7), 687-692.

Antoulas, A.C., Benner, P., \& Feng, L. (2018). Model reduction by iterative error system approximation. Mathematical and Computer Modelling of Dynamical Systems, 24(2), 103-118.

Antoulas, A.C. (2005). Approximation of large-scale dynamical systems (Vol. 6). SIAM. ISBN: 978-089871-529-3.

Antoulas, A.C. (2004). Approximation of large-scale dynamical systems: an overview. IFAC Proceedings Volumes, 37(11), 19-28.

Benner, P., \& Schneider, A. (2010, July). Balanced truncation model order reduction for LTI systems with many inputs or outputs. In Proceedings of the 19th international symposium on Mathematical Theory of Networks and Systems-MTNS (Vol. 5, pp. 1971-1974). Budapest, Hungary.

Bistritz, Y., \& Langholz, G. (1979). Model reduction by Chebyshev polynomial techniques. IEEE Transactions on Automatic Control, 24(5), 741-747. 
International Journal of Mathematical, Engineering and Management Sciences

Vol. 5, No. 5, 939-956, 2020

https://doi.org/10.33889/IJMEMS.2020.5.5.072

Bui-Thanh, T., Willcox, K., \& Ghattas, O. (2008). Parametric reduced-order models for probabilistic analysis of unsteady aerodynamic applications. AIAA Journal, 46(10), 2520-2529.

Cao, X., Saltik, M.B., \& Weiland, S. (2019). Optimal Hankel norm model reduction for discrete-time descriptor systems. Journal of the Franklin Institute, 356(7), 4124-4143.

Chahlaoui, Y., \& Van Dooren, P. (2002). A collection of Benchmark examples for model reduction of linear time invariant dynamical systems. SLICOT Working Notes.

Chahlaoui, Y., \& Van Dooren, P. (2005). Benchmark examples for model reduction of linear time-invariant dynamical systems. In Dimension Reduction of Large-Scale Systems (pp. 379-392). Springer, Berlin, Heidelberg.

Chaturvedi, D.K. (2018). Modeling and simulation of systems using MATLAB and Simulink. CRC press. London, New York.

Chidambara, M.R. (1969). Two simple techniques for the simplification of large dynamic systems. In Joint Automatic Control Conference (No. 7, pp. 669-674). IEEE. Doi: 10.1109/JACC.1969.4169310.

Daraghmeh, A., Hartmann, C., \& Qatanani, N. (2019). Balanced model reduction of linear systems with nonzero initial conditions: singular perturbation approximation. Applied Mathematics and Computation, 353, 295-307.

Daraghmeh, A., \& Qatanani, N. (2018). Error bound for non-zero initial condition using the singular perturbation approximation method. Mathematics, 6(11), 232.

Datta, B.N. (2004). Numerical methods for linear control systems (Vol. 1). Academic Press. London, New York.

Davison, E. (1966). A method for simplifying linear dynamic systems. IEEE Transactions on Automatic Control, 11(1), 93-101.

Fernando, K., \& Nicholson, H. (1982a). Singular perturbational model reduction of balanced systems. IEEE Transactions on Automatic Control, 27(2), 466-468.

Fernando, K., \& Nicholson, H. (1982b). Singular perturbational model reduction in the frequency domain. IEEE Transactions on Automatic Control, 27(4), 969-970.

Fernando, K., \& Nicholson, H. (1983). Singular perturbational approximations for discrete-time balanced systems. IEEE Transactions on Automatic Control, 28(2), 240-242.

Gajic, Z., \& Lelic, M. (2001). Improvement of system order reduction via balancing using the method of singular perturbations. Automatica, 37(11), 1859-1865.

Gawronski, W., \& Juang, J.N. (1990). Model reduction in limited time and frequency intervals. International Journal of Systems Science, 21(2), 349-376.

Glover, K. (1984). All optimal Hankel-norm approximations of linear multivariable systems and their L, $\infty-$ error bounds. International Journal of Control, 39(6), 1115-1193.

Gugercin, S. (2008). An iterative SVD-Krylov based method for model reduction of large-scale dynamical systems. Linear Algebra and its Applications, 428(8-9), 1964-1986.

Gugercin, S., \& Antoulas, A.C. (2004). A survey of model reduction by balanced truncation and some new results. International Journal of Control, 77(8), 748-766.

Guiver, C. (2019). The generalised singular perturbation approximation for bounded real and positive real control systems. Mathematical Control \& Related Fields, 9(2), 313-350.

Gupta, A.K., Kumar, D., \& Samuel, P. (2019). A mixed-method for order reduction of linear time invariant systems using big bang-big crunch and eigen spectrum algorithm. International Journal of Automation and Control, 13(2), 158-175. 
International Journal of Mathematical, Engineering and Management Sciences

Vol. 5, No. 5, 939-956, 2020

https://doi.org/10.33889/IJMEMS.2020.5.5.072

Prasad R., Pant, A.K. \& Pal, J. (1987). Model order reduction using modified truncation. National System Conference (pp.22-24). REC Kurukshetra.

Hutton, M., \& Friedland, B. (1975). Routh approximations for reducing order of linear, time-invariant systems. IEEE Transactions on Automatic Control, 20(3), 329-337.

Hwang, C., \& Wang, K.Y. (1984). Optimal Routh approximations for continuous-time systems. International Journal of Systems Science, 15(3), 249-259.

Imran, M., Ghafoor, A., \& Sreeram, V. (2014). A frequency weighted model order reduction technique and error bounds. Automatica, 50(12), 3304-3309.

Kokotovic, P.V., O'Malley Jr, R.E., \& Sannuti, P. (1976). Singular perturbations and order reduction in control theory - an overview. Automatica, 12(2), 123-132.

Kumar, D., Tiwari, J.P., \& Nagar, S.K. (2012). Reducing order of large-scale systems by extended balanced singular perturbation approximation. International Journal of Automation and Control, 6(1), 21-38.

Kumar, J., Sikander, A., Mehrotra, M., \& Parmar, G. (2019). A new soft computing approach for order diminution of interval system. International Journal of System Assurance Engineering and Management, 1-8. Doi: 10.1007/s13198-019-00865-y.

Lal, M., Singh, H., \& Parthasarathy, R. (1975). A minimal canonical realization algorithm for impulse response matrix using moments. Proceedings of the IEEE, 63(3), 538-540.

Lall, S., Marsden, J.E., \& Glavaški, S. (2002). A subspace approach to balanced truncation for model reduction of nonlinear control systems. International Journal of Robust and Nonlinear Control, 12(6), 519-535.

Liu, Y., \& Anderson, B.D. (1989). Singular perturbation approximation of balanced systems. International Journal of Control, 50(4), 1379-1405.

Lucas, T.N. (1986, November). Linear system reduction by the modified factor division method. In IEE Proceedings D (Control Theory and Applications) (Vol. 133, No. 6, pp. 293-296). IET Digital Library.

Moore, B. (1981). Principal component analysis in linear systems: controllability, observability, and model reduction. IEEE Transactions on Automatic Control, 26(1), 17-32.

Narwal, A., \& Prasad, B.R. (2016). A novel order reduction approach for LTI systems using cuckoo search optimization and stability equation. IETE Journal of Research, 62(2), 154-163.

Pal, J. (1983). Improved Pade approximants using stability equation method. Electronics Letters, 19(11), 426-427.

Pal, J. (1979). Stable reduced-order Padé approximants using the Routh-Hurwitz array. Electronics Letters, 15(8), 225-226.

Pal, J., \& Ray, L.M. (1980). Stable Pade approximants to multivariable systems using a mixed method. Proceedings of the IEEE, 68(1), 176-178.

Parmar, G., Mukherjee, S., \& Prasad, R. (2007). System reduction using factor division algorithm and eigen spectrum analysis. Applied Mathematical Modelling, 31(11), 2542-2552.

Parthasarathy, R., \& Jayasimha, K.N. (1982). System reduction using stability-equation method and modified Cauer continued fraction. Proceedings of the IEEE, 70(10), 1234-1236.

Parthasarathy, R., \& Singh, H. (1975). On suboptimal linear system reduction. Proceedings of the IEEE, 63(11), 1610-1611.

Pernebo, L., \& Silverman, L. (1982). Model reduction via balanced state space representations. IEEE Transactions on Automatic Control, 27(2), 382-387. 
International Journal of Mathematical, Engineering and Management Sciences

Vol. 5, No. 5, 939-956, 2020

https://doi.org/10.33889/IJMEMS.2020.5.5.072

Prajapati, A.K., \& Prasad, R. (2019a). Model order reduction by using the balanced truncation and factor division methods. IETE Journal of Research, 65(6), 827-842.

Prajapati, A.K., \& Prasad, R. (2019b). Reduced-order modelling of lti systems by using routh approximation and factor division methods. Circuits, Systems, and Signal Processing, 38(7), 3340-3355.

Qin, Z., \& Cheng, C.K. (2003, January). RCLK-VJ network reduction with Hurwitz polynomial approximation. In Proceedings of the 2003 Asia and South Pacific Design Automation Conference (pp. 283-291). Doi: 10.1145/1119772.1119827.

Suman, S.K., \& Kumar, A. (2019). Investigation and reduction of large-scale dynamical systems. Wseas Transactions on Systems, 18(23), 175-180.

Safonov, M.G., \& Chiang, R.Y. (1989). A Schur method for balanced-truncation model reduction. IEEE Transactions on Automatic Control, 34(7), 729-733.

Sambariya, D.K., \& Sharma, O. (2016). Routh approximation: an approach of model order reduction in siso and mimo systems. Indonesian Journal of Electrical Engineering and Computer Science, 2(3), 486-500.

Sandberg, H., \& Rantzer, A. (2004). Balanced truncation of linear time-varying systems. IEEE Transactions on Automatic Control, 49(2), 217-229.

Segalman, D.J. (2007). Model reduction of systems with localized nonlinearities. Journal of Computational and Nonlinear Dynamics, 2(3), 249-266.

Shamash, Y. (1974). Stable reduced-order models using Padé-type approximations. IEEE transactions on Automatic Control, 19(5), 615-616.

Shamash, Y. (1975). Multivariable system reduction via modal methods and Padé approximation. IEEE Transactions on Automatic Control, 20(6), 815-817.

Shamash, Y. (1981). Truncation method of reduction: a viable alternative. Electronics Letters, 17(2), 97-99.

Sikander, A., \& Prasad, R. (2015). Soft computing approach for model order reduction of linear time invariant systems. Circuits, Systems, and Signal Processing, 34(11), 3471-3487.

Sikander, A., \& Prasad, R. (2017). New technique for system simplification using Cuckoo search and ESA. Sādhanā, 42(9), 1453-1458.

Singh, N., Prasad, R., \& Gupta, H.O. (2008). Reduction of power system model using balanced realization, Routh and Padé approximation methods. International Journal of Modelling and Simulation, 28(1), 57 63.

Singh, V., Chandra, D., \& Kar, H. (2004). Improved Routh-Pade/spl acute/approximants: a computer-aided approach. IEEE Transactions on Automatic Control, 49(2), 292-296.

Singh, V.P., Chaubey, P., \& Chandra, D. (2012, March). Model order reduction of continuous time systems using pole clustering and Chebyshev polynomials. In 2012 Students Conference on Engineering and Systems (pp. 1-4). IEEE. Uttar Pradesh, India.

Tiwari, S.K., \& Kaur, G. (2018). Enhanced order reduction method for linear system using substructure preservation with dominant pole retention. International Journal of Pure and Applied Mathematics, 119(15), 2809-2823.

Wan, B.W. (1981). Linear model reduction using Mihailov criterion and Pade approximation technique. International Journal of Control, 33(6), 1073-1089.

Original content of this work is copyright ( $\odot$ International Journal of Mathematical, Engineering and Management Sciences. Uses under the Creative Commons Attribution 4.0 International (CC BY 4.0) license at https://creativecommons.org/licenses/by/4.0/ 